\title{
Influence of preparation conditions on superconducting properties of Bi-2223 thin films
}

\author{
N T MUA ${ }^{1,2}$, A SUNDARESAN ${ }^{3, *}$, N K MAN ${ }^{2}$ and D D DUNG ${ }^{2}$ \\ ${ }^{1}$ Police Department of Fire Prevention Fire Fighting and Rescue, No. 2, DinhLe Road, Hoan Kiem District, Hanoi, Vietnam \\ ${ }^{2}$ International Training Institute for Materials Science (ITIMS), Hanoi University of Science and Technology, No. 1, Dai \\ Co Viet Road, Hanoi, Vietnam \\ ${ }^{3}$ Chemistry and Physics of Materials Unit and International Centre for Materials Science, Jawaharlal Nehru Centre for \\ Advanced Scientific Research, Bangalore 560 064, India
}

MS received 12 October 2012; revised 7 December 2012

\begin{abstract}
We report electrical transport properties of $\mathrm{Bi}_{2} \mathrm{Sr}_{2} \mathrm{Ca}_{2} \mathrm{Cu}_{3} \mathrm{O}_{10+x}(\mathrm{Bi}-2223)$ superconducting thin films fabricated by pulsed-laser deposition on $\mathrm{SrTiO}_{3}$ substrate. The aim of the study was to investigate the influence of preparation conditions such as deposition temperature $\left(T_{\mathrm{S}}\right)$, annealing time $\left(t_{\mathrm{A}}\right)$ and deposition rate $(r)$. A critical temperature $\left(T_{\mathrm{c}}\right)$ as high as $110 \mathrm{~K}$ and critical current density $\left(J_{\mathrm{c}}\right)$ of $6.2 \times 10^{6} \mathrm{~A} / \mathrm{cm}^{2}$ at $20 \mathrm{~K}$ were obtained for $T_{\mathrm{S}}=$ $760^{\circ} \mathrm{C}, t_{\mathrm{A}}=4 \mathrm{~h}$ and $r=1.5 \AA / \mathrm{s}$. We also investigated the effect of Li doping on Bi-2223 thin films. Li intercalation results in high resistive onset transition temperature and the resistivity shows broadening in magnetic field that increases with field. The large broadening of resistivity curve in magnetic field suggests that this phenomenon is directly related to the intrinsic superconducting properties of the copper oxide superconductors. The sudden drop in $J_{c}$ at relatively low magnetic field $(H<0.5$ tesla) is due to the effect of Josephson weak-links at the grain boundaries.
\end{abstract}

Keywords. Bi-2223; thin film; pulsed laser deposition; critical current density.

\section{Introduction}

From both scientific and technology points of view, it is important to study how the superconducting transition temperature changes with thickness of film, substrate and also to examine critical thickness for the occurrence of superconducting states. In the case of high-temperature superconductors (HTS), the dimensionality has also been an interesting subject for understanding the mechanism of superconducting phenomenon. One of the important materials that has been investigated extensively is $\mathrm{Bi}_{2} \mathrm{Sr}_{2} \mathrm{Ca}_{2} \mathrm{Cu}_{3} \mathrm{O}_{10+x}$ (Bi-2223) superconducting thin film (Simon 2003). Several fabrication techniques such as pulsed-laser deposition (PLD) (Dijkkamp et al 1987), molecular beam epitaxy (LPE) (Fujino et al 2001), co-evaporation (Cui et al 2001), metal organic chemical vapour deposition (MOCVD) (Doudkowsky et al 1997) have been used to prepare Bi-2223 films. Among these methods, PLD method is known to be suitable for fabricating films with complex stoichiometry.

Thin films of Bi-cuprates fabricated by a pulsed laser deposition (PLD) method show promising superconducting properties. Applying this technique, one could obtain thin

*Author for correspondence (sundaresan@jncasr.ac.in) films of single-phase Bi-2212 and Bi-2223. However, it was found that fabrication of $\mathrm{Bi}-2223$ thin films was more difficult than that of $\mathrm{Bi}-2212$. Critical temperatures of 69 and $78 \mathrm{~K}$ were obtained for as-deposited $\mathrm{Bi}-2212$ and $\mathrm{Bi}$ 2223 , respectively at the deposition temperatures of 820 and $850{ }^{\circ} \mathrm{C}$ (Norihisa et al 1999), though a better crystalline film was obtained at a lower temperature of $750{ }^{\circ} \mathrm{C}$ for $\mathrm{Bi}-2212$ and $\mathrm{Bi}-2223$. The substrate temperature was also found to influence surface morphology of the film. $\mathrm{Bi}_{2} \mathrm{Sr}_{2} \mathrm{Ca}_{n-1} \mathrm{Cu}_{n} \mathrm{O}_{2(n+2)+\delta}(n=1,2,3)$ thin films are prepared in general on vicinal $\mathrm{SrTiO}_{3}$ substrates by pulsed-laser deposition. Optimization of substrate temperature, laser fluence and post-annealing conditions produces single-phase, welloriented and smooth films. On (0 001$) \mathrm{SrTiO}_{3}$ substrates, $c$ axis oriented Bi-2212 films with $J_{\mathrm{c}}=2 \times 10^{6} \mathrm{~A} / \mathrm{cm}^{2}$ at $60 \mathrm{~K}$ and $T_{\mathrm{c}, \text { zero }}=82 \mathrm{~K}$ are obtained (Rössler et al 2001). Bi-2223 film grown at a temperature of $725^{\circ} \mathrm{C}$ displays a sharp transition of $10 \mathrm{~K}$, which is close to the best result $(11 \mathrm{~K})$ obtained by Ishii and Hatano (2000) and $13 \mathrm{~K}$ obtained by Matthiesen et al (1991).

Further, several studies of the effect of Li intercalation (Kambe et al 1996, 1998) on the nominal composition of $\mathrm{Bi}-2223$ have been reported, where it was observed that increase in the quantity of lithium led to the reduction of the volume fraction of $\mathrm{Bi}-2223$ phase. On the other hand, 
transport measurements have shown that the increase of lithium content leads to the improvement in transport critical current density, $J_{\text {ct }}$ (Mihalache et al 2001). The systematic studies of $\mathrm{LiF}$ (Mihalache et al 2003a, b) and $\mathrm{LiCl}$ (Minh et al 2003) doped Bi-2223 systems revealed that a small quantity of lithium compound helps in the

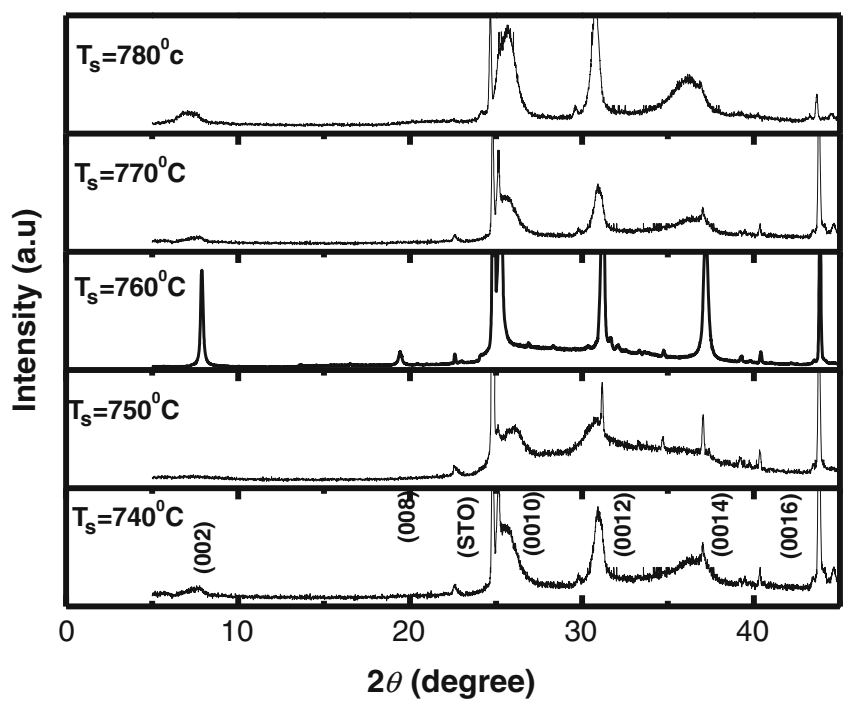

Figure 1. XRD patterns of $\mathrm{Bi}-2223$ thin films on $\mathrm{SrTiO}_{3}$ substrate deposited at various substrate temperatures: 740, 750, 760, 770 and $780{ }^{\circ} \mathrm{C}$. formation of high- $T_{\mathrm{c}}$ superconducting phase Bi-2223. The transport and intergrain critical current densities $\left(J_{\mathrm{ct}}\right.$ and $J_{\mathrm{cj}}$ ) vs the doping level exhibit a maximum and the critical temperature $T_{\mathrm{c}}$ of $110 \mathrm{~K}$ superconducting phase Bi2223 reaches $120 \mathrm{~K}$ (Mihalache et al 2001, 2003a, b).

In this paper, we report effect of preparation conditions on the properties of $\mathrm{Bi}_{2} \mathrm{Sr}_{2} \mathrm{Ca}_{2} \mathrm{Cu}_{3} \mathrm{O}_{10+x}$ thin films prepared by the PLD method using BSCCO target which was prepared by the solid-state reaction method.

\section{Experimental}

The target was prepared from the starting materials, $\mathrm{Bi}_{2} \mathrm{O}_{3}$, $\mathrm{SrCO}_{3}, \mathrm{CaCO}_{3}$ and $\mathrm{CuO}$, by the solid-state reaction method. The stoichiometric amount of these oxide powders were mixed thoroughly and calcined at $840{ }^{\circ} \mathrm{C}$ for $24 \mathrm{~h}$ in air. This step was repeated at $850{ }^{\circ} \mathrm{C}$ for $72 \mathrm{~h}$ in air with several intermittent grindings to improve the homogeneity. The calcined powder was compressed into a disk of $15 \mathrm{~mm}$ in diameter and thickness of $4 \mathrm{~mm}$ with the use of polyvinyl alcohol as a binder. The compacted powder disk was heat-treated at $860{ }^{\circ} \mathrm{C}$ for $24 \mathrm{~h}$. Before deposition, (100) $\mathrm{SrTiO}_{3}$ (STO) substrates were annealed in air at $1000{ }^{\circ} \mathrm{C}$ for $1 \mathrm{~h}$. The excimer laser $(\lambda=248 \mathrm{~nm})$ was operated at $300 \mathrm{~mJ} /$ pulse for $30 \mathrm{~min}$ for each deposition. Substrate was mounted on the heater in the cross-area of PLD plume caused by the beam and its temperature $\left(T_{\mathrm{s}}\right)$ was in the range of $740-780{ }^{\circ} \mathrm{C}$.
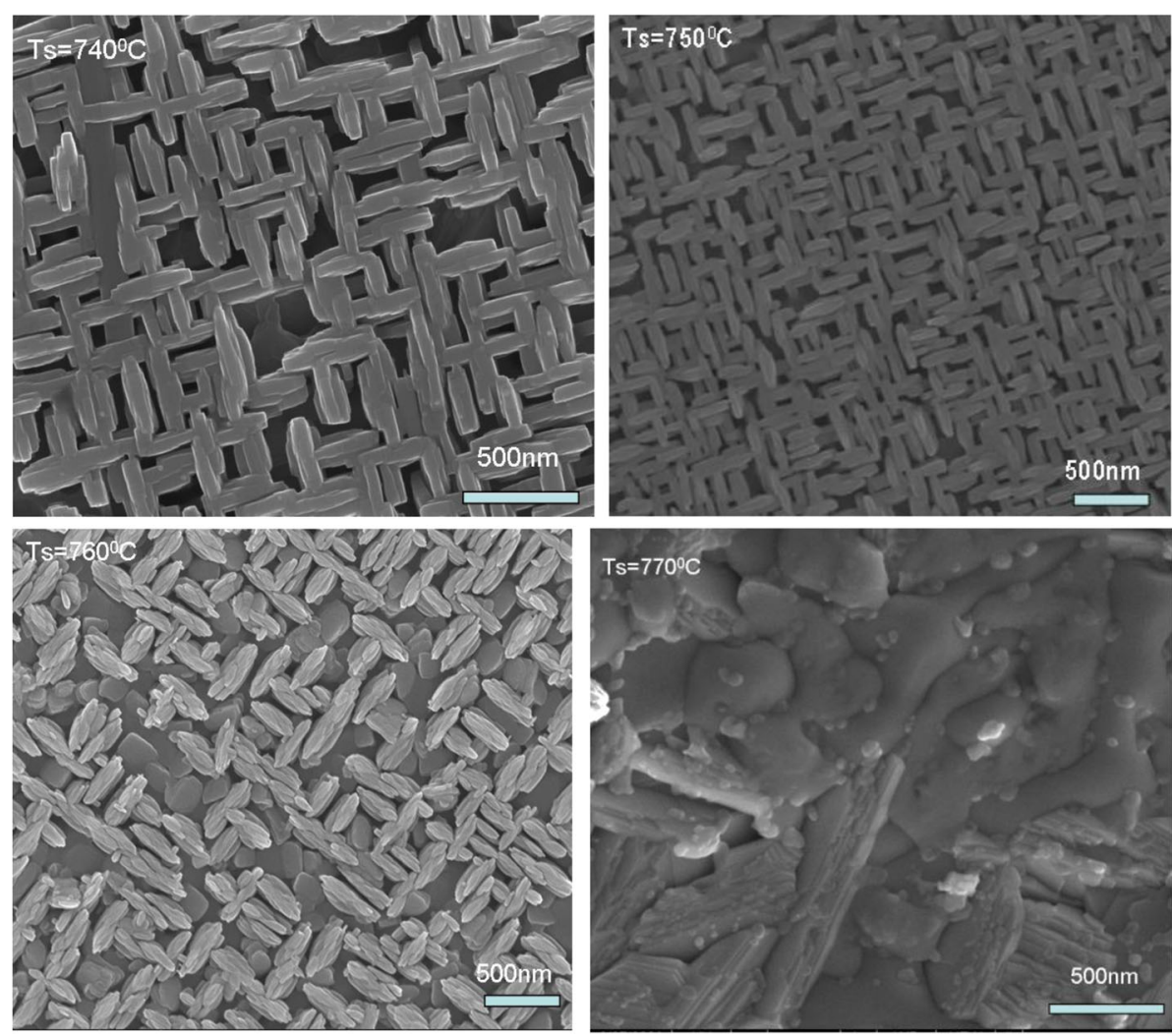

Figure 2. SEM micrograph of Bi-2223 films prepared at various temperatures. 
The oxygen pressure was kept at $0 \cdot 25$ mbar. The films were annealed at $680^{\circ} \mathrm{C}$ in the range of $1-5 \mathrm{~h}$ and cooled down to room temperature in oxygen pressure of $1000 \mathrm{mbar}$. The film thickness was about of $300 \mathrm{~nm}$. To compare the superconducting properties, we prepared $\mathrm{Bi}-2223$ films with various amounts of $\mathrm{Li}$ intercalation. The same preparation conditions were followed for $\mathrm{Li}$ intercalated films.

D.C. electrical resistance measurements were carried out using the standard four-probe method with silver alloy solders as electrical contacts. The superconducting transition temperature $\left(T_{\mathrm{c}}\right)$ was determined as the midpoint between 90 and $10 \%$ of the resistive transition. The structure and surface morphology of the films were studied by X-ray diffraction and field emission scanning electron microscope (FESEM), respectively. Magnetic measurements were carried out using the vibrating sample magnetometer option in the physical property measuring system (PPMS, quantum design). The critical current density $J_{\mathrm{c}}$ of the film was calculated by Bean model

$$
J_{\mathrm{c}}=60 a|\Delta M| / b(3 a-b),
$$

where $a$ and $b$ are the length and width $(a>b$, in $\mathrm{cm}$ ) of the sample plane perpendicular to the applied magnetic field. $\Delta M$ is the difference of the magnetization $\left(\mathrm{emu} / \mathrm{cm}^{3}\right)$ between the field-up and field-down branches.

\section{Results and discussion}

\subsection{Properties of Bi-2223 thin film on STO substrate}

X-ray diffraction (XRD) pattern for Bi-2223 thin films at various substrate temperatures are shown in figure 1 . The presence of (002) peak in XRD pattern of thin films corresponds to a well-crystallized orthorhombic phase with $c$-axis

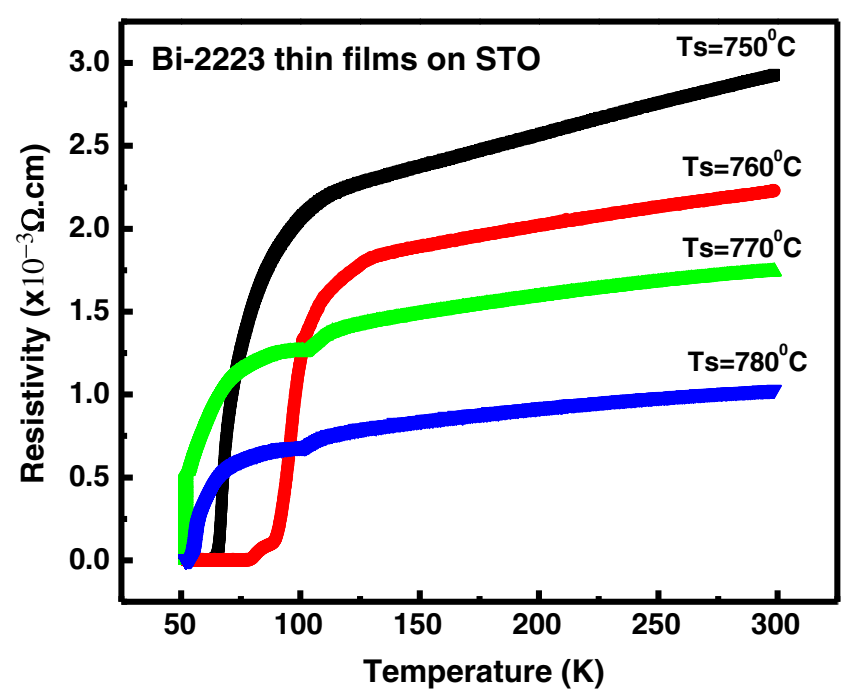

Figure 3. Resistivity of Bi-2223 thin films at different substrate temperatures.

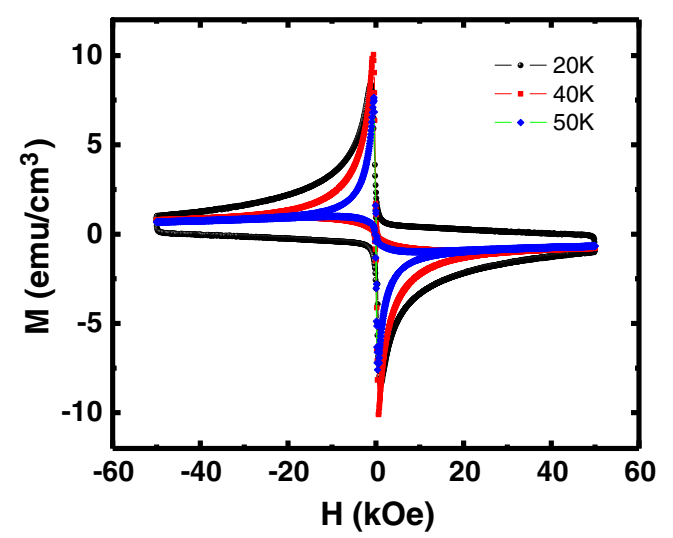

Figure 4. Magnetic field dependence of current density at different temperatures.

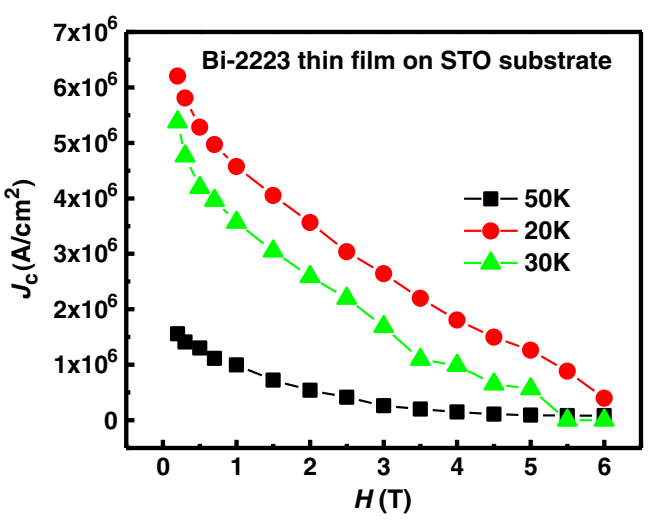

Figure 5. $\mathrm{M}-\mathrm{H}$ of $\mathrm{Bi}-2223$ thin films at 20, 30 and $50 \mathrm{~K}$.

oriented perpendicular to the film surface. The obtained value for $c$-axis length is for the film deposited at substrate temperature of $760{ }^{\circ} \mathrm{C}, 37.68 \AA$ which is comparable to that reported in the literature. This confirms that Bi-2223 thin films grown on single crystalline (100) STO substrate is of high structural quality. This also reflects perfection of orientation of different $c$-axis oriented blocks of the film which is relatively normal to the substrate. It is observed that there exists a trace amount of $\mathrm{CuO}$ as a secondary phase. Images of scanning electron micrographs of Bi-2223 films prepared at different temperatures are shown in figure 2. It can be clearly seen that there are particles with Bi-2223 composition on the surface of all the films. Many grain boundaries and voids could be seen for the films on STO due to large lattice mismatch $(+2.31 \%)$. Relatively, smooth films with a surface roughness, grain size of around $200 \mathrm{~nm}$ and a low particulate density were obtained on $\mathrm{SrTiO}_{3}$ as seen in figure 2 .

Figure 3 shows temperature dependence of resistivity for the films at different substrate temperatures. All the films show a metallic behaviour in the normal state. A typical resistivity curve measured on ex-situ annealed films, as shown in 

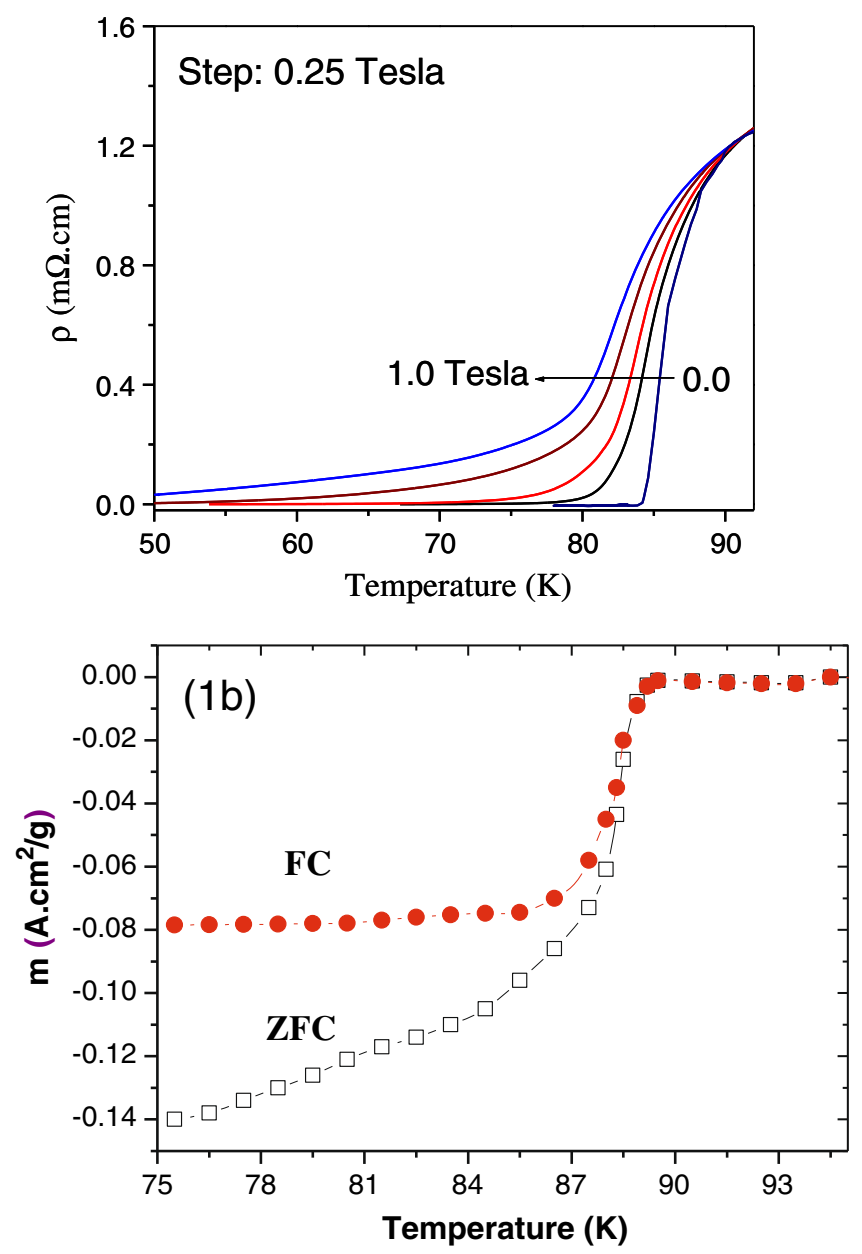

Figure 6. $\rho$ vs $T$ of BSCCO thin films for different magnetic fields and FC and ZFC of BSCCO thin films measurement at $H=$ 100 Oe.
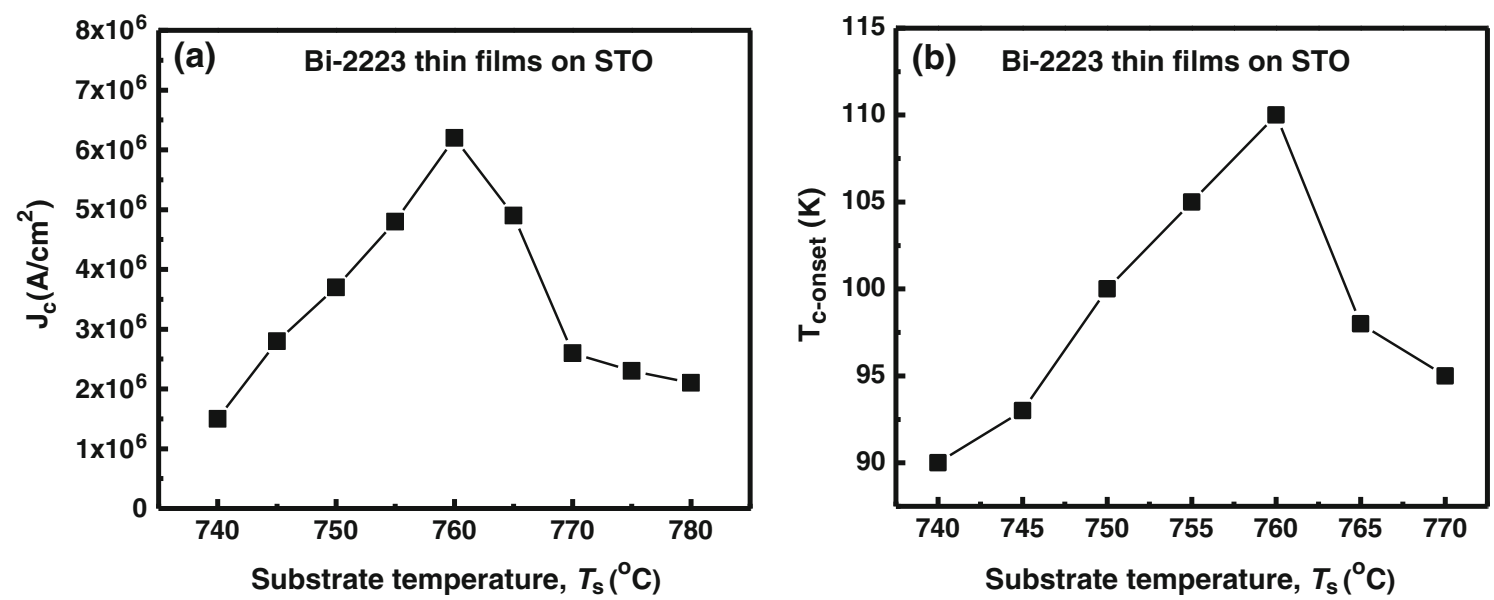

Figure 7. Effect of deposition temperature $\left(T_{\mathrm{S}}\right)$ on $J_{\mathrm{c}}$ and $T_{\mathrm{c}}$ in Bi-2223 thin films.

figure 3, displays a broad transition with a transition temperature, $T_{\mathrm{c}-\text { onset }}=110 \mathrm{~K}$ and zero resistance at $95 \mathrm{~K}$. The broad transition may be due to the presence of $\mathrm{CuO}$ impurity. The resistivity tail is very short compared with the width of transition. This could be due to the presence of inhomogeneities in the oxidation state of grains of superconducting materials of good structural quality. It has been reported that the critical temperature of $\mathrm{Bi}-2223$ materials is very 
much dependent on the oxygen concentration. Since oxygen incorporation depends sensitively on the deposition conditions, slight changes of the conditions during growth could generate variations in oxygen concentration. In particular, we would like to point out that growing superconductor layer induces change in the infrared reflectivity of the substrate film system, which could result in a shift in substrate temperature. It is interesting to notice that the resulting inhomogeneities in oxygen concentration would lead to variations in the structural parameters.

The critical current density at various temperatures and magnetic field obtained from the analysis of the magnetization data (figure 4) using Bean model is shown in figure 5. The value of $J_{c}$ at $20 \mathrm{~K}$ and $0.5 \mathrm{~T}$ is $6.2 \times 10^{6} \mathrm{~A} / \mathrm{cm}^{2}$ that decreases with increasing magnetic field and temperature. In this configuration, Lorenz force is perpendicular to the $a b$ plane and vortices induced by the applied magnetic field have to cross the superconducting $\mathrm{CuO}_{2}$ planes for flux flow to occur. It is shown that $J_{\mathrm{c}}$ decreases rapidly when the field is applied parallel to $c$-axis of the film. The sudden drop in $J_{\mathrm{c}}$ as soon as a magnetic field is applied $(H<0.5 \mathrm{~T})$ is due to the effect of Josephson weak links at the grain boundaries (Minh

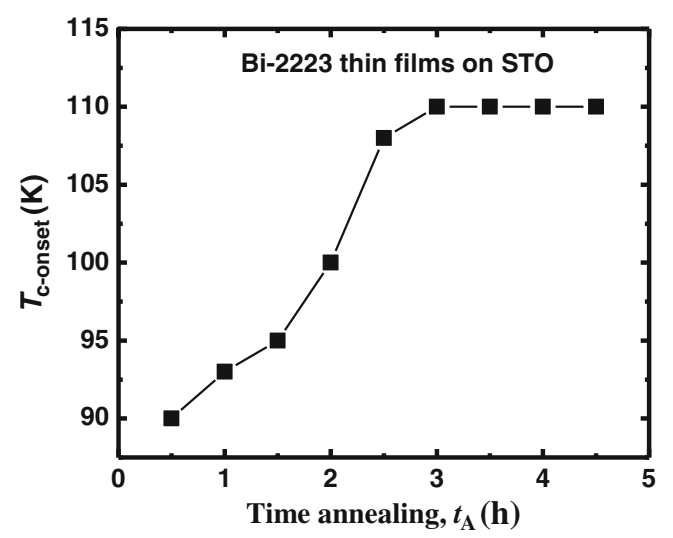

Figure 8. Effect of annealing time $\left(t_{\mathrm{A}}\right)$ on $T_{\mathrm{c}}$ in $\mathrm{Bi}-2223$ thin films.

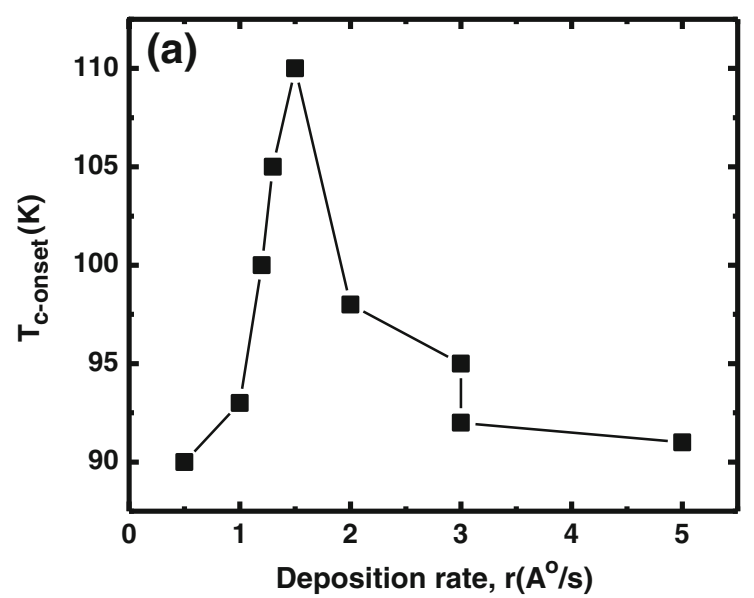

et al 2003). At higher applied magnetic field $(H>0.5 T), J_{\mathrm{c}}$ decreases slowly due to the flux pinning inside the grains. From the critical current density, $J_{\mathrm{c}}$, the pinning force density $F_{\mathrm{P}}$ can be calculated by using the equation:

$$
F_{\mathrm{P}}=J_{\mathrm{c}} \times H .
$$

Resistivity as a function of temperature at different magnetic fields up to 1 Tesla is shown in figure 6(a). The results can be summarized as follows: (i) At zero field, the transition is sharp and has a zero-resistance temperature, $T_{\mathrm{c} 0}$ of $90 \mathrm{~K}$; (ii) the resistive transition in the presence of the magnetic field exhibits a broadening, which increases with increasing magnetic field. The large broadening of resistivity curve in magnetic field suggests that this phenomenon is directly related to the intrinsic superconducting properties of this kind of oxide superconductors. The resistivity in the normal state, above $T_{\mathrm{c}}$, shows a strong para-conducting behaviour. The magnetization data of BSCCO thin film obtained under zero-field cooling (ZFC) and field-cooling (FC) processes are shown in figure 6(b). It is shown that the diamagnetic transition temperature $\left(T_{\mathrm{c}}\right)$ of BSCCO thin film is $95 \mathrm{~K}$. We calculated $H_{\mathrm{c} 2}$ from the formula:

$$
H_{\mathrm{C} 2}=\sqrt{ } 2 \kappa H_{\mathrm{C}}, H_{\mathrm{c} 2} \sim \kappa H_{\mathrm{c}} ; \kappa=0,8,
$$

in which

$$
H_{\mathrm{C}}=\left[\frac{2}{\mu_{0}}\left(g_{\mathrm{n}}-g_{\mathrm{s}}\right)\right]^{\frac{1}{2}} \text {. }
$$

\subsection{Effect of deposition temperature $\left(T_{\mathrm{S}}\right)$}

Heating of the substrate during deposition was required to provide thermal energy for the deposited atoms to migrate on the surface and to arrange themselves in the energetically favoured lattice. A higher substrate temperature $\left(T_{\mathrm{s}}\right)$ can provide activation energy for adatoms to occupy the position of potential minima and to increase surface and volume diffusion enhancing recrystallization due to the coalescence

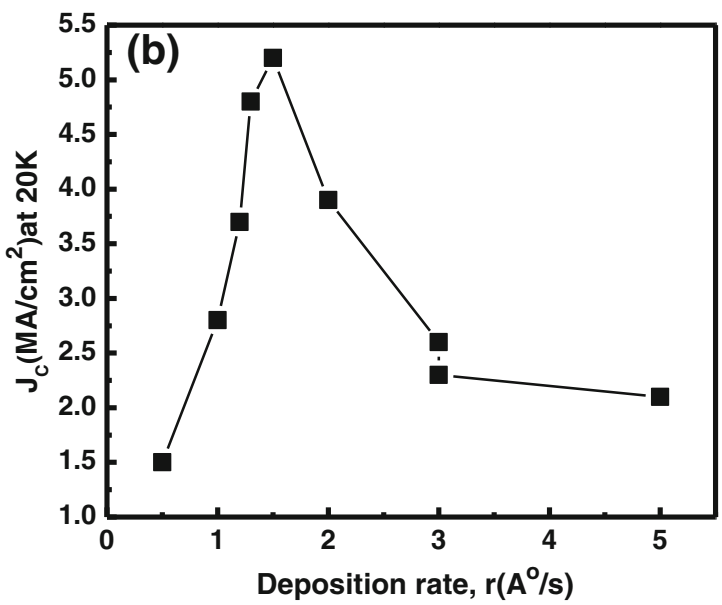

Figure 9. Effect of deposition rate $(r)$ on $T_{\mathrm{c}}(\mathrm{a})$ and $J_{\mathrm{c}}$ at $20 \mathrm{~K}$ (b) of Bi-2223 thin films on STO substrate. 


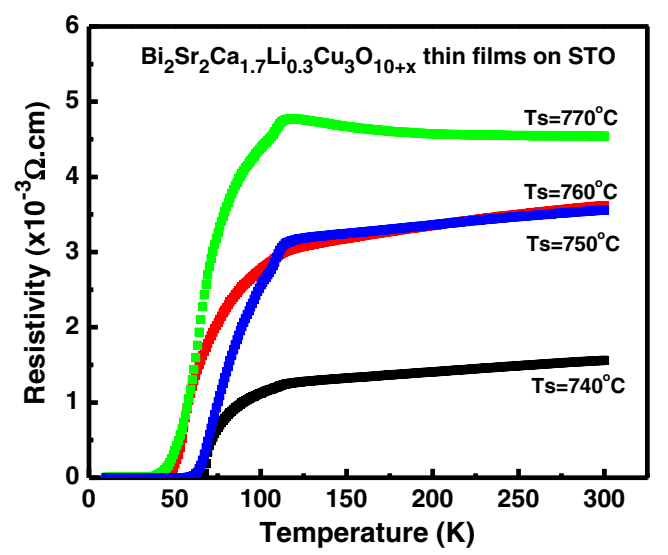

Figure 10. Temperature dependent resistivity of Li-intercalated Bi-2223 thin films prepared on $\mathrm{SrTiO}_{3}$ substrate at different substrate temperatures.

of islands. On the other hand, higher $T_{\mathrm{S}}$ could cause a serious interdiffusion between film and substrate, which could degrade superconducting properties of the films.

In the present study, effects of $T_{\mathrm{s}}$ on properties of Bi-2223 thin films have been investigated, while the other parameters were kept constant: time of annealing, $t_{\mathrm{A}}=3 \mathrm{~h}$ and deposition rate, $r=2 \AA / s$. As shown in figure 7, the temperature region between 740 and $780{ }^{\circ} \mathrm{C}$ provides an optimum superconducting transition temperature $\left(T_{\mathrm{c}}\right)$ and critical current density $\left(J_{\mathrm{c}}\right)$. These temperatures are high enough to allow sufficient surface migration and interdiffusion of the atoms for the formation of the desired orthorhombic Bi-2223 crystal structure. It shows that the optimal transition temperature, $T_{\mathrm{c}}=110 \mathrm{~K}$, was obtained at $T_{\mathrm{s}}$ of around $760{ }^{\circ} \mathrm{C}$, where the highest critical current density, $J_{\mathrm{c}}=5.2 \times 10^{6} \mathrm{~A} / \mathrm{cm}^{2}$ at $20 \mathrm{~K}$ was obtained.

\subsection{Effect of annealing time $\left(t_{\mathrm{A}}\right)$}

It is well known that the superconductivity of Bi-2223 is strongly affected by oxygen content. The annealing at $680^{\circ} \mathrm{C}$ in oxygen is believed to be important for controlling oxygen content of the films. Figure 8 shows transition temperature of Bi-2223 films as a function of annealing time. After $3 \mathrm{~h}$ of annealing, the optimal critical temperature of $110 \mathrm{~K}$ is reached. Further annealing does not influence the transition temperature and oxygen content, $5 \mathrm{~h}$ of reduction treatment, the value is same $3 \mathrm{~h}$.

\subsection{Effect of deposition rate ( $r)$}

Figure 9 is the dependence of $T_{\mathrm{c}}$ and $J_{\mathrm{c}}$ as a function of Bi2223 deposition rate $\left(T_{\mathrm{s}}=760^{\circ} \mathrm{C}, t_{\mathrm{A}}=3 \mathrm{~h}\right)$. These results showed that the optimal deposition rate is $1.5 \AA / \mathrm{s}$. At lower deposition rate, the films' surface was characterized by voids between grains. At higher deposition rate, outgrowths appear which increase in number with further increase of deposition rate. The outgrowth has deleterious effect on $T_{\mathrm{c}}$ and $J_{\mathrm{c}}$ of Bi-2223 films.

\subsection{Effect of Li intercalation in Bi-2223 thin films}

Figure 10 depicts typical temperature dependence of electrical resistivity of Li substituted $\mathrm{Bi}-2223$. A similar metallic trend in the normal state was obtained. Li intercalation results in a considerable increase in $T_{\mathrm{c}-\text { onset }}(115 \mathrm{~K})$. It is also well known that Li-doping lowers melting temperature of this phase, so the decrease in oxygen content would be expected. It can be seen that a linear decrease of resistivity at lower substrate temperatures produces a sharp drop with narrow transition. It should be mentioned that as $\mathrm{Li}$ concentration increased, the normal state resistivity of sample decreases and zero-resistivity temperature increases.

\section{Conclusions}

Bi-2223 thin films of good quality with perfect $c$-axis orientation have critical temperature of $110 \mathrm{~K}$ and critical current density of $6.2 \times 10^{6} \mathrm{~A} / \mathrm{cm}^{2}$ at $20 \mathrm{~K}$ in $0.5 \mathrm{~T}$. The critical temperature is increased to $115 \mathrm{~K}$ for $\mathrm{Li}$ intercalated $\mathrm{Bi}-2223$ thin films. The resistive transition in the presence of magnetic field exhibits a broadening induced by the magnetic field and the broadening increases with increasing field. The sudden drop in $J_{\mathrm{c}}$ at a relatively lower magnetic field is applied $(H<0.5 \mathrm{~T})$ due to the effect of Josephson weak-links at the grain boundaries. These films have a sufficiently good quality to make the high-performance microwave components. Considering the flux-pinning behaviour, critical current density strongly depends on the applied magnetic field. Large critical current values are obtained in high magnetic fields which are of interest for applications.

\section{Acknowledgments}

We thank the International Centre for Materials Science, Jawaharlal Nehru Centre for Advanced Scientific Research (JNCASR), Bangalore, for providing a visiting fellowship. This work was partially supported by the Nafosted Program under the Project No.103.02-2010.22. Authors thank Prof CNR Rao for helpful discussion and providing facilities required for this study.

\section{References}

Cui X et al 2001 Physica C 351, 75.

Dijkkamp D, Venkatesan T, Wu X D, Shaheen S A, Jisrawi N, MinLee Y H, McLean W L and Croft M 1987 Appl. Phys. Lett. 619 51

Doudkowsky M, Santiso J, Berton S, Figueras A and Bassas J 1997 Chem. Phys. $\mathbf{2 8 8} 1$

Fujino H, Kasai Y, Ota H, Migita S, Yamamori H, Matsumoto K and Sakai S 2001 Physica C 362256 
Ishii A and Hatano T 2000 Physica C 340173

Kambe S, Guo Y G, Dou S X, Liu H K and Wakahara Y 1996 Proceedings of IPMM'97 2451

Kambe S, Guo Y G, Liu H K, Wakahara Y, Maeda H, Kakimoto K and Yavuz M 1998 Supercond. Sci. Technol. 11 1061

Matthiesen M M, Graybeal J M, Orlando T P and Vander Sande J B 1991 IEEE Trans. Magn. 1223272

Mihalache V, Aldica G, Giusca C and Miu L 2001 J. Supercond. Inc. Novel Magn. 14575
Mihalache V, Aldica G, Popa S and Crisan 2003a Physica C 384 451

Mihalache V, Aldica G, Popa S and Miu D 2003b J. Supercond. Inc. Novel Magn. 16869

Minh N D, Hien T D, Man N K, Truong D V and So P V 2003 Commun. Phys. 13142

Norihisa A, Shinohara K, Matsunami N and Takai Y 1999 Supercond. Sci. Technol. 20312

Rössler R, Pedarnig J D and Jooss C 2001 Physica C 36121

Simon R 2003 RF military electronics (RF design - August) 28. 